\title{
East North Central States Census Division
}

National Cancer Institute

\section{Source}

National Cancer Institute. East North Central States Census Division. NCI Thesaurus. Code $C 76340$.

A census division of the United States consisting of Illinois, Indiana, Michigan, Ohio, and Wisconsin. 